\title{
Morphological Characteristics of Korean Dried Ginseng Products
}

\author{
Hee-Do Hong ${ }^{1 *}$, Chang-Won Cho ${ }^{1}$, Young-Chan Kim ${ }^{1}$, Eunyoung Kim ${ }^{1}$, Young-Kyung Rhee ${ }^{1}$, \\ Jeonghae Rho', and Seung-Hoe Choi ${ }^{2}$ \\ ${ }^{1}$ Korea Food Research Institute, Seongnam 463-420, Korea \\ ${ }^{2}$ Department of General Studies, School of Liberal Arts and Sciences, Korea Aerospace University, Goyang 412-791, Korea
}

The purpose of this study was to determine a standard quality characteristic through the evaluation and statistical analysis of the morphological characteristics of dried ginseng (white ginseng) products. Samples of 4-year-old 30 and 50 piece grade curved ginseng and 5-year-old 25 and 15 piece grade straight ginseng products were collected from a market, with 5 to 10 packs of each product being collected annually over a 5-year period (2006-2010). Morphological characteristics, such as weight, length, diameter, and surface color, were measured and statistically analyzed to present a standard quality characteristic value using mean $\pm 3 \mathrm{SD}$, a range that excluded outlier. The 4-year-old curved ginseng samples of 50 and 30 piece grade were 4.80 to $6.12 \mathrm{~cm}$ and 5.28 to 7.60 $\mathrm{cm}$ long, 0.22 to $1.70 \mathrm{~cm}$ and 0.21 to $2.07 \mathrm{~cm}$ wide, and weighed 5.28 to $7.40 \mathrm{~g}$ and 8.62 to $12.26 \mathrm{~g}$, respectively. The 5-year-old straight ginseng samples of 25 and 15 piece grade were 9.66 to $15.47 \mathrm{~cm}$ and 10.66 to $16.80 \mathrm{~cm}$ long, 1.32 to $1.94 \mathrm{~cm}$ and 1.48 to 2.43 $\mathrm{cm}$ wide, and weighed 9.18 to $16.40 \mathrm{~g}$ and 15.89 to $24.82 \mathrm{~g}$, respectively. The surface color of the different piece grades in the same type of dried ginseng product was similar, whereas the straight ginseng demonstrated a lower level of brightness, but the relative redness and yellowness were of higher levels, than that of curved ginseng.

Keywords: Panax ginseng, Dried ginseng products, Piece grade, Morphological characteristics, Standardization

\section{INTRODUCTION}

Ginseng (Panax ginseng Meyer) is a perennial plant in the Araliaceae family and the Panax genus and mainly cultivated in Northeast Asia, including Korea. In general, 4- to 6-year-old roots have been utilized as an important medicine or functional food ingredient [1]. Much research has been conducted on ginseng both in Korea and internationally to identify various physiological activities, including immune-enhancing functions and anti-fatigue effects, which have been attributed to such molecules as phenolic compounds, polysaccharides, polyacetylene, and alkaloids, in addition to saponin, a major active substance $[2,3]$. However, few studies focus on the appearance and morphological characteristics unique to

(c) This is an Open Access article distributed under the terms of the Creative Commons Attribution Non-Commercial License (http://creativecommons.org/licenses/by-nc/3.0/) which permits unrestricted non-commercial use, distribution, and reproduction in any medium, provided the original work is properly cited. ginseng. In the late 1990s, some studies on the morphological characteristics of ginseng were reported, but these mostly included samples collected during a short period of time, with only the color, individual weight, lateral root and rhizome development being compared to those parameters of foreign ginseng or other species. Furthermore, mainly fresh ginseng or red ginseng were used as the samples $[4,5]$. Currently, the domestic ginseng products distributed on the Korean market can be classified into several categories, including fresh ginseng, red ginseng, and dried ginseng, depending on the manufacturing method. Fresh ginseng accounted for $45 \%$, or the largest share, of all the products consumed, and red ginseng

Received 18 Jan. 2012, Revised 02 Apr. 2012, Accepted 02 Apr. 2012

*Corresponding author

E-mail: honghd@kfri.re.kr

Tel: +82-31-780-9285, Fax: +82-31-709-9876 
products captured $33 \%$ of the market share, followed by dried ginseng products at 20\% [6]. Dried ginseng refers to ginseng products that have not been steamed but dried in sunlight, hot-air or by other methods. These products are classified as 4-, 5-, and 6-year-old roots based on the cultivation period and as straight ginseng, curved ginseng, and half-curved ginseng based on the shape of the root. The quality is classified into $1 \mathrm{st}, 2 \mathrm{nd}$, and 3rd grade, depending on the condition of the product, such as ratio of cracked or damaged roots or by the external color. In addition, package units are divided into $5,8,10,15,20$, $25,30,40,50,60$, and 75 pieces (number of pieces per $300 \mathrm{~g}$ package) for each grade based on the individual weight or size.

Currently, it is difficult to standardize or maintain the quality of dried ginseng products that are packaged and sold by processing type, quality grade, or piece grade by size. In addition, the current grades or quality standards should be based on scientific evidence. Although the Ginseng Industry Act and associated regulations specify the diameter and weight as quality indices for the classification of shapes and piece grades, scientific evidence in support of these standards is lacking.

Therefore, in this study, we sampled curved and straight ginseng products of 4-year-old 50 and 30 piecegrade and 5-year-old 15 and 25 piece-grade sizes, respectively, among the dried ginseng products that are distributed throughout Korea. Dried ginseng samples were collected for 5 years, and their morphological characteristics were measured and statistically analyzed to determine the standard quality characteristics per grade and also to provide basic data for establishing practical future quality standards for dried ginseng products.

\section{MATERIALS AND METHODS}

\section{Sample}

The dried ginseng products included 4-year-old, 1st- grade, curved ginseng of 30 and 50 piece grades and 5 -year-old, 1st-grade, straight ginseng of 15 and 25 piece grades produced between 2006 and 2010. A total of 40 products, 5 to 10 products per year, were collected from major domestic dried ginseng markets, including Seoul Gyeongdong Market and those in the Chungnam Geumsan ginseng market and used as the samples for the analysis of the morphological characteristics. The dried ginseng products were all packaged and certified by the inspection office of National Agricultural Cooperative Federation or manufacturer. The samples collected each year are shown in Table 1.

\section{Morphological properties Length}

A total of 10 pieces of dried ginseng were randomly selected from the same package, and the length from the tip of the rhizome to the longest lateral root was measured. An average value was obtained and represented the body length $(\mathrm{cm})$ of the relevant sample.

\section{Diameter}

A total of 10 pieces of dried ginseng were randomly selected from the same package, and the diameter of the thickest portion of the rhizome was measured using calipers. An average value was obtained and represented the diameter $(\mathrm{cm})$ of the relevant sample.

\section{Weight}

A total of 10 pieces of dried ginseng were randomly selected from the same package, and the weights of the individual pieces were measured. An average value was obtained and represented the average weight $(\mathrm{g})$ of the relevant sample.

\section{Surface color}

A total of 5 pieces of dried ginseng were randomly selected from the same package, and the surface color

Table 1. Sample collection

\begin{tabular}{|c|c|c|c|c|}
\hline \multirow{2}{*}{ Production year } & \multicolumn{2}{|c|}{ Curved ginseng (piece) ${ }^{1)}$} & \multicolumn{2}{|c|}{ Straight ginseng (piece) ${ }^{2)}$} \\
\hline & $30^{3)}$ & 50 & 15 & 25 \\
\hline 2006 & 10 & 10 & 10 & 10 \\
\hline 2007 & 10 & 10 & 10 & 10 \\
\hline 2008 & 10 & 10 & 10 & 10 \\
\hline 2009 & 8 & 8 & 8 & 8 \\
\hline 2010 & 8 & 8 & 8 & 8 \\
\hline
\end{tabular}

\footnotetext{
${ }^{1)}$ Four-year-old, 1st grade.

${ }^{2)}$ Five-year-old, 1st grade.

${ }^{3)}$ Piece grade: number of pieces per $300 \mathrm{~g}$ package for each dried ginseng products.
} 
of the rhizome was measured 3 times using a colorimeter (Chroma Meter CR 21; Minolta, Osaka, Japan). An average value was obtained to represent the surface color for brightness (L), redness (a) and yellowness (b).

\section{Statistical analysis}

The morphological characteristics per piece grade of each dried ginseng product were analyzed using Minitab ver. 14 (Minitab Inc., Pittsburgh, PA, USA) to obtain the normality, mean, standard deviation (SD), median, and

Table 2. Statistical analysis of the morphological characteristics of curved ginseng (4-year-old, 50 piece grade)

\begin{tabular}{|c|c|c|c|c|c|c|}
\hline & & & & & urface col & \\
\hline & Total length $(\mathrm{cm})$ & Diameter (cm) & Weight (g) & Brightness & Redness & Yellowness \\
\hline K-S statistic ${ }^{1)}$ & 0.063 & 0.225 & 0.079 & 0.146 & 0.073 & 0.093 \\
\hline$p$-value ${ }^{2)}$ & 0.150 & 0.010 & 0.150 & 0.030 & 0.150 & 0.150 \\
\hline Mean & 5.491 & 0.963 & 6.344 & 74.492 & 2.654 & 25.849 \\
\hline Sta Dev & 0.229 & 0.247 & 0.353 & 3.512 & 0.819 & 2.255 \\
\hline Skewness $^{3)}$ & 0.310 & 0.470 & 0.270 & 0.840 & 0.150 & -0.410 \\
\hline Kurtosis $^{4)}$ & 0.600 & -1.450 & -0.060 & 0.050 & -0.580 & -0.140 \\
\hline Median & 5.470 & 0.890 & 6.340 & 73.580 & 2.540 & 26.200 \\
\hline Maximum & 6.117 & 1.360 & 7.200 & 83.803 & 4.550 & 30.314 \\
\hline Minimum & 4.940 & 0.630 & 5.520 & 69.500 & 1.275 & 20.446 \\
\hline mean $+3 \mathrm{SD}$ & 6.177 & 1.703 & 7.404 & 85.028 & 5.111 & 32.614 \\
\hline
\end{tabular}

1) The Kolmogorov-Smirnoff (K-S) statistic $D_{n}$ is defined as: $D_{n}=\max \left[\left|F_{n}(x)-F(x)\right|\right]$ where $D_{n}$ is know as the K-S distance. $\mathrm{n}=$ total number of data points, $\mathrm{F}(\mathrm{x})=$ distribution function of the fitted distribution. $F_{n}(x)=i / n$ i=the cumulative rank of the data point.

2) The $p$-value measures consistency by calculating the probability of observing the results from your sample of data or a sample with results more extreme, assuming the null hypothesis is true. The smaller the $p$-value, the greater the inconsistency.

${ }^{3)}$ A measure of symmetry, or more precisely, the lack of symmetry.

${ }^{4)}$ A measure of whether the data are peaked or flat relative to a normal distribution.

5) Outliers are data outside of mean-3SD and mean+3SD.

Table 3. Statistical analysis of the morphological characteristics of curved ginseng (4-year-old, 30 piece grade)

\begin{tabular}{|c|c|c|c|c|c|c|}
\hline & \multirow{2}{*}{ Total length $(\mathrm{cm})$} & \multirow{2}{*}{ Diameter $(\mathrm{cm})$} & \multirow{2}{*}{ Weight (g) } & \multicolumn{3}{|c|}{ Surface color } \\
\hline & & & & Brightness & Redness & Yellowness \\
\hline K-S statistic ${ }^{1)}$ & 0.076 & 0.209 & 0.108 & 0.077 & 0.058 & 0.103 \\
\hline$p$-value ${ }^{2)}$ & 0.150 & 0.010 & 0.150 & 0.150 & 0.150 & 0.150 \\
\hline Mean & 6.438 & 1.140 & 10.442 & 73.553 & 2.658 & 25.969 \\
\hline Sta Dev & 0.387 & 0.309 & 0.606 & 3.056 & 0.692 & 2.082 \\
\hline Skewness $^{3)}$ & -0.140 & 0.580 & 0.130 & 0.180 & -0.080 & -0.590 \\
\hline Kurtosis ${ }^{4)}$ & -0.140 & -1.300 & -1.080 & -0.170 & -0.370 & -0.180 \\
\hline Median & 6.460 & 0.980 & 10.350 & 73.150 & 2.590 & 26.310 \\
\hline Maximum & 7.260 & 1.761 & 11.670 & 80.891 & 4.043 & 29.366 \\
\hline Minimum & 5.600 & 0.780 & 9.370 & 66.420 & 1.100 & 20.814 \\
\hline mean-3SD ${ }^{5)}$ & 5.276 & 0.212 & 8.624 & 64.385 & 0.582 & 19.723 \\
\hline mean $+3 \mathrm{SD}$ & 7.600 & 2.068 & 12.260 & 82.721 & 4.734 & 32.215 \\
\hline
\end{tabular}

1) The Kolmogorov-Smirnoff (K-S) statistic $D_{n}$ is defined as: $D_{n}=\max \left[\left|F_{n}(x)-F(x)\right|\right]$ where $D_{n}$ is know as the K-S distance. n=total number of data points, $F(x)=$ distribution function of the fitted distribution. $F_{n}(x)=i / n$ i=the cumulative rank of the data point.

${ }^{2)}$ The $p$-value measures consistency by calculating the probability of observing the results from your sample of data or a sample with results more extreme, assuming the null hypothesis is true. The smaller the $p$-value, the greater the inconsistency.

${ }^{3)}$ A measure of symmetry, or more precisely, the lack of symmetry.

${ }^{4)}$ A measure of whether the data are peaked or flat relative to a normal distribution.

${ }^{5)}$ Outliers are data outside of mean-3SD and mean+3SD. 
outlier range of the dried ginseng measurements [7]; the values of the standard quality characteristics were acquired on the basis of mean $\pm 3 \mathrm{SD}$

\section{RESULTS AND DISCUSSION}

\section{Morphological characteristics of curved ginseng (4-year-old) by piece grade}

The length, diameter, weight and surface color of
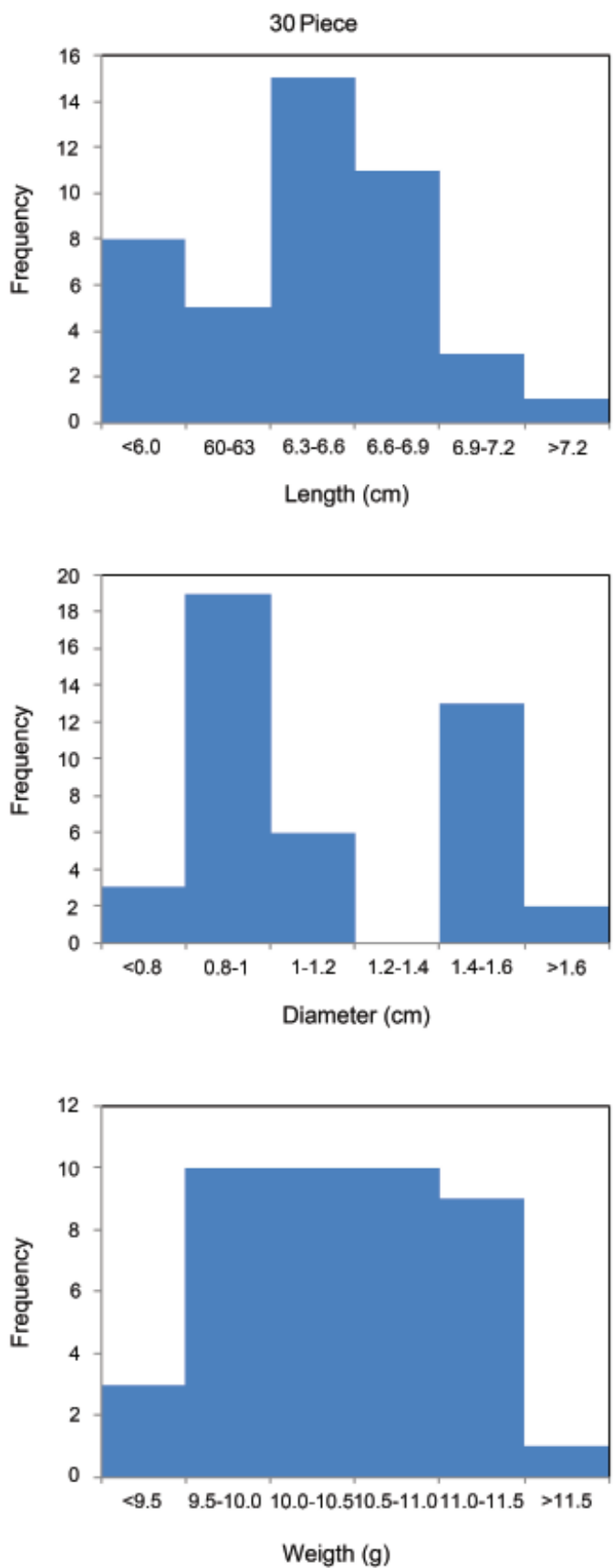

the 4-year-old curved ginseng (1st grade) products collected during the 5-year period, with 46 parts each from the 30-piece and 50-piece packages (the category most widely consumed) were measured. The statistics for each measurement are shown in Tables 2 and 3, and the interval frequency of length, diameter, and weight are shown in Fig. 1.

For the overall length, the mean and the median were 6.44 and $6.46 \mathrm{~cm}$ and 5.49 and $5.47 \mathrm{~cm}$ for the 30 and
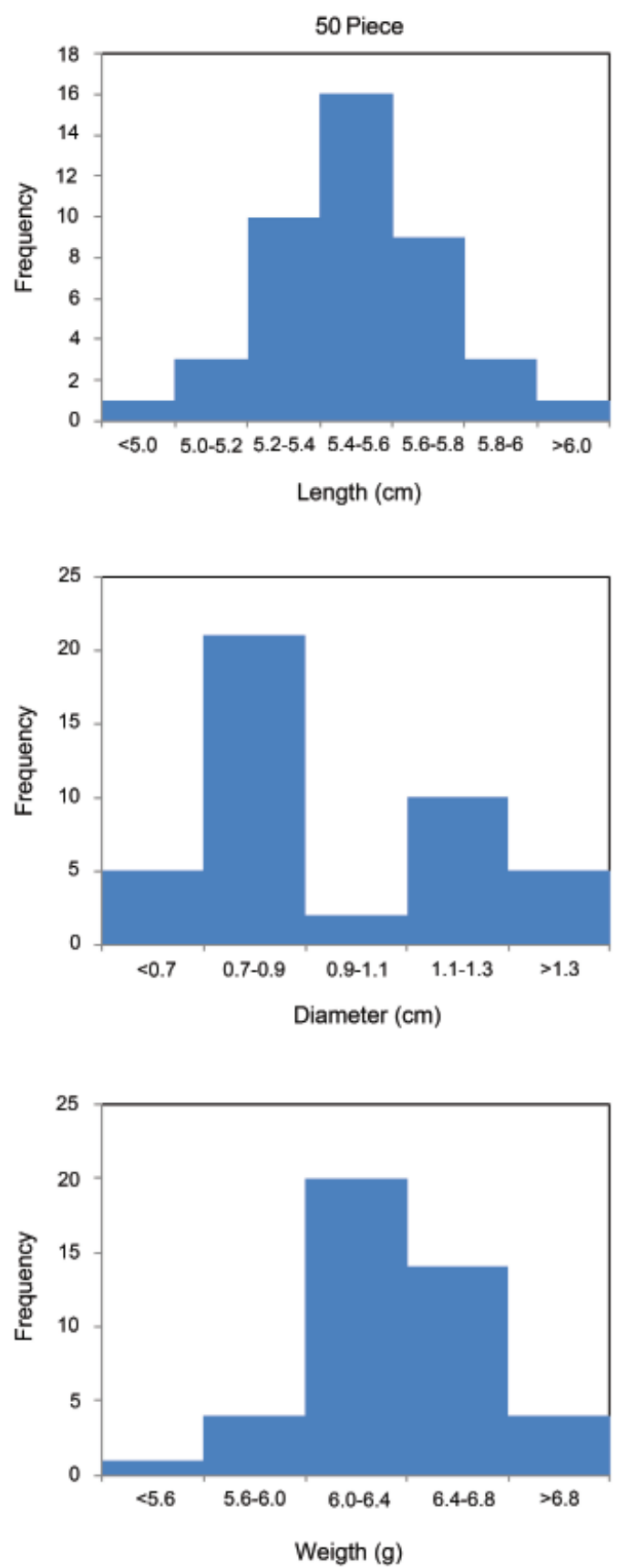

Fig. 1. Interval distribution of morphological characteristics for curved ginseng in different package units. 
50 piece grades, respectively, and the overall length intervals, based on the minimum and maximum values, were 5.60 to $7.26 \mathrm{~cm}$ and 4.94 to $6.11 \mathrm{~cm}$, respectively. The standard deviations, showing the deviation within the same piece grade, were 0.39 for the 30 piece grade and 0.23 for the 50 piece grade. For the distribution of length by interval, there was a certain portion of overlap between under $6.0 \mathrm{~cm}$ for the 30 piece grade and over $6.0 \mathrm{~cm}$ for the 50 piece grade; however, the difference in length between piece-grade was, in general, clearly defined. The 30 piece grade showed a trend of negative skew values for the distribution of the overall length, skewing slightly to the left compared with the median, and exhibited a kurtosis, which represents the shape of the center, of a small negative value, with a gentle normal distribution. In contrast, the 50 piece grade displayed a skew of 0.310 and a kurtosis of 0.600 , which was generally skewed to the right, compared with the median and appeared normal distribution, with a peak in the center. The mean and the median of the diameter of the 30 and 50 piece-grade ginseng samples were 1.14 and $0.98 \mathrm{~cm}$ and 0.96 and $0.89 \mathrm{~cm}$, respectively. The diameter interval, as based on the minimum and maximum values, was 0.78 to $1.76 \mathrm{~cm}$ for the 30 piece grade and 0.63 to 1.36 $\mathrm{cm}$ for the 50 piece grade. The standard deviations for the diameter were 0.31 for the 30 piece grade and 0.25 for the 50 piece grade, which were slightly higher when compared to those for the length. The normality of the diameter indicated that both the 30 and 50 piece grades were similarly skewed, but the kurtosis values but did not demonstrate similar normality. The means and the medians of the weights were 10.44 and $9.92 \mathrm{~g}$ for the 30 piece grade and 6.34 and $6.34 \mathrm{~g}$, for the 50 piece grade, respectively. The weight intervals, as based on the minimum and maximum values, were 9.37 to $11.67 \mathrm{~g}$ for the 30 piece grade and 5.52 to $7.20 \mathrm{~g}$ for the 50 piece grade, showing relatively distinct differences by piece grade when compared to the length or diameter. The standard deviation was 0.60 for the 30 piece grade and 0.35 for the 50 piece grade, showing little individual differences within the same piece grade. In terms of distribution, both the 30 and 50 piece-grade samples tended to skew slightly to the right compared to the median, and the distribution of the median showed normality, with slight kurtosis.

Color according to the existing dried ginseng quality index has been described in rather vague terms, which are not scientific. Therefore, the surface color of the dried ginseng in this study was measured using a colorimeter and then statistically analyzed to standardize the measurement using objective values. The color was measured in terms of $\mathrm{L}, \mathrm{a}$, and $\mathrm{b}$. The means and the medians of 30 piece grade were 73.55 and 73.15 for brightness, 2.66 and 2.59 for redness, 25.97 and 26.31 for yellowness and the standard deviations were $3.01,0.69$, and 2.08 , respectively. The means and the medians of 50 piece grade

Table 4. Statistical analysis of the morphological characteristics of straight ginseng (5-year-old, 15 piece grade)

\begin{tabular}{|c|c|c|c|c|c|c|}
\hline & \multirow{2}{*}{ Total length $(\mathrm{cm})$} & \multirow{2}{*}{ Diameter $(\mathrm{cm})$} & \multirow{2}{*}{ Weight $(\mathrm{g})$} & \multicolumn{3}{|c|}{ Surface color } \\
\hline & & & & Brightness & Redness & Yellowness \\
\hline K-S statistic ${ }^{1)}$ & 0.771 & 0.076 & 0.079 & 0.098 & 0.068 & 0.100 \\
\hline$p$-value ${ }^{2)}$ & 0.590 & 0.150 & 0.150 & 0.150 & 0.150 & 0.150 \\
\hline Mean & 13.730 & 1.936 & 20.356 & 66.039 & 4.799 & 30.215 \\
\hline Sta Dev & 1.024 & 0.163 & 1.488 & 3.750 & 1.167 & 2.749 \\
\hline Skewness ${ }^{3)}$ & 0.520 & 0.810 & -0.170 & 0.370 & -0.320 & -0.600 \\
\hline Kurtosis ${ }^{4)}$ & -0.180 & 0.930 & -0.140 & -0.670 & -0.340 & 0.000 \\
\hline Median & 13.520 & 1.920 & 20.515 & 65.565 & 4.835 & 30.345 \\
\hline Maximum & 16.248 & 2.460 & 23.580 & 74.349 & 7.170 & 38.855 \\
\hline Minimum & 11.930 & 1.680 & 16.720 & 59.820 & 2.246 & 22.384 \\
\hline mean-3SD ${ }^{5)}$ & 10.658 & 1.447 & 15.892 & 54.789 & 1.298 & 21.968 \\
\hline mean $+3 \mathrm{SD}$ & 16.802 & 2.425 & 24.820 & 77.289 & 8.300 & 38.462 \\
\hline
\end{tabular}

1) The Kolmogorov-Smirnoff (K-S) statistic $D_{n}$ is defined as: $D_{n}=\max \left[\left|F_{n}(x)-F(x)\right|\right]$ where $D_{n}$ is know as the K-S distance. $\mathrm{n}=$ total number of data points, $\mathrm{F}(\mathrm{x})=$ distribution function of the fitted distribution. $F_{n}(x)=i / n \mathrm{i}=$ the cumulative rank of the data point.

${ }^{2)}$ The $p$-value measures consistency by calculating the probability of observing the results from your sample of data or a sample with results more extreme, assuming the null hypothesis is true. The smaller the $p$-value, the greater the inconsistency.

${ }^{3)}$ A measure of symmetry, or more precisely, the lack of symmetry.

4) A measure of whether the data are peaked or flat relative to a normal distribution.

${ }^{5)}$ Outliers are data outside of mean-3SD and mean+3SD. 
Table 5. Statistical analysis of the morphological characteristics of straight ginseng (5-year-old, 25 piece grade)

\begin{tabular}{|c|c|c|c|c|c|c|}
\hline & \multirow{2}{*}{ Total length $(\mathrm{cm})$} & \multirow{2}{*}{ Diameter $(\mathrm{cm})$} & \multirow{2}{*}{ Weight (g) } & \multicolumn{3}{|c|}{ Surface color } \\
\hline & & & & Brightness & Redness & Yellowness \\
\hline K-S statistic ${ }^{1)}$ & 0.084 & 0.072 & 0.153 & 0.113 & 0.130 & 0.087 \\
\hline$p$-value ${ }^{2)}$ & 0.150 & 0.150 & 0.033 & 0.150 & 0.104 & 0.150 \\
\hline Mean & 12.551 & 1.633 & 12.787 & 66.849 & 4.627 & 29.453 \\
\hline Sta Dev & 0.965 & 0.103 & 1.201 & 4.073 & 1.102 & 3.226 \\
\hline Skewness ${ }^{3)}$ & -0.370 & -0.270 & 1.010 & -0.240 & -0.570 & 0.300 \\
\hline Kurtosis ${ }^{4)}$ & 0.160 & -0.180 & 0.930 & -0.560 & -0.490 & -1.010 \\
\hline Median & 12.690 & 16.350 & 12.555 & 66.900 & 4.855 & 29.235 \\
\hline Maximum & 14.740 & 1.854 & 15.900 & 73.747 & 6.270 & 35.193 \\
\hline Minimum & 10.470 & 1.391 & 10.584 & 57.960 & 2.220 & 24.520 \\
\hline mean-3SD & 9.656 & 1.326 & 9.184 & 54.630 & 1.321 & 19.775 \\
\hline mean $+3 \mathrm{SD}$ & 15.446 & 1.941 & 16.390 & 79.068 & 7.933 & 39.131 \\
\hline
\end{tabular}

1) The Kolmogorov-Smirnoff (K-S) statistic $D_{n}$ is defined as: $D_{n}=\max \left[\left|F_{n}(x)-F(x)\right|\right]$ where $D_{n}$ is know as the K-S distance. n=total number of data points, $\mathrm{F}(\mathrm{x})=$ distribution function of the fitted distribution. $F_{n}(x)=i / n$ i=the cumulative rank of the data point.

2) The $p$-value measures consistency by calculating the probability of observing the results from your sample of data or a sample with results more extreme, assuming the null hypothesis is true. The smaller the $p$-value, the greater the inconsistency.

3) A measure of symmetry, or more precisely, the lack of symmetry.

4) A measure of whether the data are peaked or flat relative to a normal distribution.

5) Outliers are data outside of mean-3SD and mean+3SD.

were 74.49 and 73.58 for brightness, 2.65 and 2.54 for redness, 25.85 and 26.20 for yellowness, and the standard deviations were $3.51,0.89$, and 2.26 , respectively. These results indicated that the brightness varied little among individuals within the same piece grade, whereas, on the basis of the mean, the redness varied significantly among individuals.

\section{Morphological characteristics of straight ginseng (5-year-old) by piece grade}

The 5-year-old straight ginseng was produced from fresh ginseng by drying, while maintaining its original shape. Samples were collected over 5 years, with 46 parts each from the 15 and 25 piece grades; the morphological characteristics were analyzed by the same method used for the curved ginseng. The results of the statistical analysis are shown in Tables 4 and 5, and the interval frequency of the length, diameter, and weight is shown in Fig. 2.

For the overall length of the straight ginseng products, including the lateral roots, the means and the medians of the 15 piece grade were 13.73 and $13.52 \mathrm{~cm}$, respectively, and those of the 25 piece grade were 12.55 and $12.69 \mathrm{~cm}$, respectively. The overall length intervals, as based on the minimum and maximum values, were 11.93 to $16.25 \mathrm{~cm}$ for the 15 piece grade and 10.47 to 14.74 $\mathrm{cm}$ for the 25 piece grade. The standard deviations of the overall length measurements were 1.02 for the 15 piece grade and 0.97 for the 25 piece grade. For the distribution of length by interval, unlike the curved ginseng, the two piece grades of straight ginseng overlapped slightly for the 11 to $14 \mathrm{~cm}$ interval. For the distribution of the overall lengths, the 15 piece grade was skewed to the right compared with the median, and the 25 piece grade was skewed to the left, with negative values. The kurtosis for both piece grades showed small positive value, showing gentle normal distributions $(p>0.15)$. The mean and median of the diameter of the 15 and 25 piece grades were 1.94 and $1.92 \mathrm{~cm}$ and 1.63 and $1.63 \mathrm{~cm}$, respectively. The diameter intervals, as based on the minimum and maximum values, were 1.68 to $2.46 \mathrm{~cm}$ for the 15 piece grade and 1.13 to $1.25 \mathrm{~cm}$ for the 25 piece grade. The standard deviation was 0.16 for the 15 piece grade and 0.10 for the 25 piece grade. In terms of distribution, the 15 piece grade was skewed to the right, with a positive value, compared with the median, whereas the 25 piece grade was skewed to the left, with a negative value, with certain portions overlapping near the diameter of 1.7 to $1.8 \mathrm{~cm}$. The kurtosis of 15 piece-grade was 0.81 , with a higher frequency in the center, and that of the 25 piece grade showed a kurtosis of a relatively low negative value in the low center region but showed a normal distribution. The means and the medians of the weights were $20.36 \mathrm{~g}$ and $20.52 \mathrm{~g}$ for the 15 piece grade and 

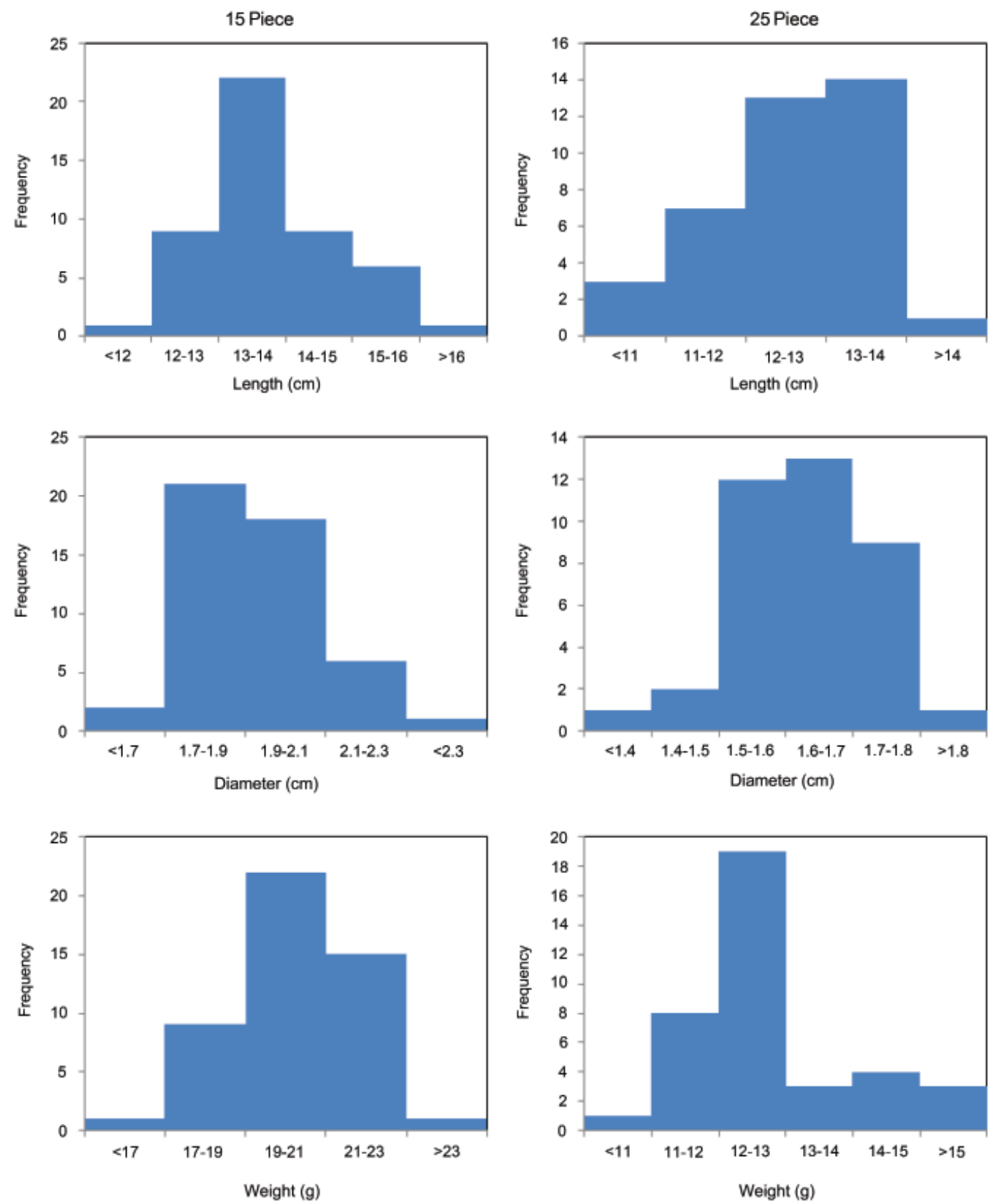

Fig. 2. Interval distribution of morphological characteristics for straight ginseng in different package units.

$12.79 \mathrm{~g}$ and $12.56 \mathrm{~g}$ for the 25 piece grade, respectively. The weight interval, based on the minimum and maximum values, was 16.72 to $23.58 \mathrm{~g}$ for the 15 piece grade and 10.58 to $15.90 \mathrm{~g}$ for the 25 piece grade, showing the most distinct difference by piece grade in comparison to the length or diameter. The standard deviation of the weight was 1.45 for the 15 piece grade and 1.20 for the 25 piece grade. For the distribution, the 15 piece grade was skewed slightly to the left compared to the median, with a rather gentle normality of the distribution, whereas the 25 piece grade showed a symmetric normality with the 15 piece grade.

The color of the 15 piece-grade straight ginseng was measured in terms of $\mathrm{L}$, a, and $\mathrm{b}$. The means and medians for the 15 piece grade were $66.04,65.57$ for brightness, 4.80 and 4.83 for redness and 30.21 and 30.35 for yellowness, respectively. The standard deviations were 3.75 , 1.16 , and 2.75 , respectively. For the 25 piece grade, L, a, and $b$ were 66.85 and 66.90, 4.63 and 4.86 and 29.45 and 29.24, respectively. The standard deviations were 4.01, 
1.10 , and 3.23, respectively. Overall, the differences in surface color among the piece grades were not large and the brightness values were lower, whereas the redness and yellowness values were rather higher when compared to those for the curved ginseng.

Although the saponin, polyphenol, and acidic polysaccharide contents have been used as quality indices to determine quality differences by species, year, and various grades of ginseng products, studies on the morphological characteristics for the standardization of ginseng samples are seldom conducted. In fact, morphological characteristics have been defined by descriptive methods in Korean and foreign ginseng-related specifications and regulations. Additionally, the number of ginseng rhizomes per package has been counted and then classified into 15, 25, 30 , and 50 piece grades when $15,25,30$, and 50 pieces are contained per $300 \mathrm{~g}$ of package, respectively. In the case of piece-grade classification, the weight index has been merely defined as 17.6 to $25.0 \mathrm{~g}, 11.1$ to $13.5 \mathrm{~g}, 8.8$ to $11.0 \mathrm{~g}$, and 5.6 to $6.7 \mathrm{~g}$, respectively, under the domestic Law for Ginseng Industry.

In this study, 30 and 50 piece-grade curved ginseng and 15 and 25 piece-grade straight ginseng samples, which are widely distributed throughout Korea, were selected. Morphological characteristic analysis and statistical analysis were conducted, and standard quality characteristic intervals were established and presented as mean $\pm 3 \mathrm{SD}$, in the range of the excluding outliers. The 30 piece-grade curved ginseng showed morphological characteristics of 5.28 to $7.60 \mathrm{~cm}$ in length, 0.21 to $2.07 \mathrm{~cm}$ in diameter, and 8.62 to $12.26 \mathrm{~g}$ in weight. The 50 piece-grade curved ginseng was 4.80 to $6.12 \mathrm{~cm}$ in length, 0.22 to $1.70 \mathrm{~cm}$ in diameter, and 5.28 to $7.40 \mathrm{~g}$ in weight. In case of the 5-year-old straight ginseng, 15 piece grade showed morphological characteristics of 10.66 to $16.80 \mathrm{~cm}$ in length, 1.48 to $2.43 \mathrm{~cm}$ in diameter and 15.89 to $24.82 \mathrm{~g}$ in weight, and the 25 piece grade showed morphological characteristics of 9.66 to 15.45 $\mathrm{cm}$ in length, 1.32 to $1.94 \mathrm{~cm}$ in diameter, and 9.18 to $16.40 \mathrm{~g}$ in weight. In particular, the weights were similar, except for the rather wide range and estimated interval, to those defined in the current ginseng-related regulations. For the surface color, it was thought that there is almost no difference according to the dried ginseng type, and standard quality characteristics by piece grade were not presented. Curved ginseng showed a brightness of 64.09 to 83.95 (mean of 74.02), redness of 0.41 to 4.91 (mean of 2.66), and yellowness of 19.44 to 32.39 (mean of 25.91); straight ginseng showed a brightness of 54.73 to 78.07 (mean of 66.47), redness of 1.3 to 8.14 (mean of 4.72), and yellowness of 20.94 to 38.82 (mean of 29.88). Among all of the morphological characteristics, the diameter, length, and redness significantly varied among individuals and tended to show overlapped intervals of quality characteristics by piece grade.

\section{REFERENCES}

1. Korea Ginseng \& Tobacco Research Institute. Korean ginseng. Daejeon: Korea Ginseng \& Tobacco Research Institute, 1994.

2. Ernst E. Panax ginseng: An Overview of the Clinical Evidence. J Ginseng Res 2010;34:259-263.

3. Vuksan V, Sievenpipper J, Jovanovski E, J. AL. Current Clinical Evidence for Korean Red Ginseng in Management of Diabetes and Vascular Disease: A Toronto's Ginseng Clinical Testing Program. J Ginseng Res 2010;34:264-273.

4. Sohn HJ, Baek NI, Lee SK, Nho KB, Kim MW. The comparison of the appearances between the Korean ginseng and the Chinese ginseng. Korean J Ginseng Sci 1997;21:187-195.

5. Chung YY, Lee MG, Chung CM, Jo JS. Comparison of plant growth and morphological characteristics among the Korean ginseng, the American ginseng and Bamboo ginseng. J Ginseng Res 1998;22:147-153.

6. Ministry for Food, Agriculture, Forestry and Fisheries. Statistics for Korean ginseng. Gwacheon: Ministry for Food, Agriculture, Forestry and Fisheries, 2009.

7. Miller I, Miller M. Mathematical statistics with application. Upper Saddle River: Prentice Hall, 2004. 